\title{
Hiperplasia Supra-Renal Congênita por Deficiência de 11-ß-Hidroxilase
}

\author{
Congenital Adrenal Hyperplasia due to 11-Beta-hydroxylase Deficiency
}

Ramires Tosatti Júnior, Haroldo Silva de Souza, Alexandre Tosatti

Hospital Geral de Goiânia e Hospital São Bernardo - Goiânia, GO - Aparecida de Goiânia, GO

Este artigo tem o objetivo de relatar o diagnóstico e a evolução clínica de um paciente de 15 anos portador de uma disfunção congênita da esteroideogênese adrenal, que pode apresentar-se como hipertensão arterial de diagnóstico muitas vezes tardio (adolescência), virilização ou formas perdedoras de sal (nascimento e infância).
The objective of this article is to relate the diagnostic and clinical evolution of a 15 year old patient with a congenital adrenal steroidogenesis dysfunction that can present as hypertension diagnosed later in life (adolescence), virilization or salt wasting (birth and childhood).
Hiperplasia adrenal congênita, devido a inabilidade de síntese de cortisol, freqüentemente apresenta-se clinicamente por sinais de excesso androgênico (masculinização da genitália externa feminina). Alguns pacientes também desenvolvem sinais e sintomas decorrentes da deficiência de aldosterona como hiponatremia, hipercalemia e hipovolemia, levando, se não tratados, ao choque e morte após algumas semanas de vida. Entretanto, somente após a década de 50, foi reconhecido que uma pequena porcentagem desses pacientes desenvolvem hipertensão, sensível a terapia glicocorticóide. Esta subpopulação era acometida por um distúrbio metabólico distinto, a deficiência de 11-ß-hidroxilase; enquanto pacientes sem hipertensão apresentavam a bem conhecida deficiência de 21-hidroxilase.

\section{Relato do Caso}

Adolescente, quinze anos, masculino, pardo, deu entrada no setor de emergência (HUGO - Hospital de Urgências de Goiânia) com quadro súbito de cefaléia parietal intensa acompanhada de tonturas, ptose palpebral e desvio de rima labial, ambos à esquerda. Constatou-se pico hipertensivo (pressão arterial de 250x160 mmHg em membro superior direito, paciente em decúbito dorsal) e realizou-se tomografia computadorizada axial de crânio, evidenciando hemorragia intraparenquimatosa e periventricular à esquerda, sem efeito de massa nas estruturas adjacentes (fig. 1).

Após medicação sintomática, foi referenciado ao setor de terapia intensiva de um hospital de apoio (Hospital São Bernardo - Aparecida de Goiânia/GO). Na sua história pregressa, pai relatava desenvolvimento infantil normal até os três anos, quando passou a apresentar ginecomastia, pubarca e axilarca precoces, além de acne aos seis anos de idade.

Exame clínico de entrada: acianótico, eupnéico, corado, hidratado, afebril. Acne em tronco (fig. 2), porção proximal de membros e face (fig. 3). Na internação na unidade de terapia intensiva, apresentava-se com pressão arterial de 190x125 mmHg (sob proteção medicamentosa de nifedipina oral) e freqüência cardíaca de 77 batimentos por minuto. Tireóide impalpável. Ictus cordis desviado para esquerda, palpável no $7^{0}$ espaço intercostal esquerdo, no nível da linha axilar anterior. Ritmo cardíaco regular, em dois tempos, com bulhas hiperfonéticas, sem sopros ou extra-sístoles. Aparelho respiratório sem alterações. Ausência de visceromegalias aparentes ou "sopros" em região abdominal. Membros inferiores sem edemas. Discreta disartria, ptose palpebral e desvio fixo de rima labial (ambos para esquerda). Tônus, força muscular, reflexos superficiais e profundos inalterados. Pupilas isocóricas e fotorreagentes. Altura: 1,85 m, Peso: 61,7 kg e IMC: 18,02. Envergadura: 1,83 m, Pé-Púbis: $94 \mathrm{~cm}$. Estágio puberal: pêlos pubianos (estágio $V$ de Tanner), genitália (estágio $\mathrm{V}$ de Tanner). Exames laboratoriais de entrada - $\mathrm{Ht}=39,8 \% ; \mathrm{Hm}=4,35 \mathrm{tera} / \mathrm{l} ; \mathrm{Hb}=14 \mathrm{~g} / \mathrm{dl}$; $\mathrm{VCM}=91 \mathrm{fl}, \mathrm{HCM}=32,18 p g ; \mathrm{CHCM}=35,18 \mathrm{~g} / \mathrm{dl}$; RDW = 14\%. Leuco: $6500 \mathrm{MI} / \mathrm{ME} / \mathrm{BA} / \mathrm{SEG}$ 0/0/1/62 BAS/EOS/ LINF/MON 0/5/26/6; U =60; $\mathrm{Cr}=1,3 ; \mathrm{Na}^{+}=136 ; \mathrm{K}^{+}$ $=3,0 ; \mathrm{Ca}^{++}=10,6 ; \mathrm{Mg}^{++}=2,4 ; \mathrm{TGO}=31 ; \mathrm{TGP}$ $=27 ; \mathrm{TP}=12,5 \mathrm{~s} ; \mathrm{AP}=100 \%$; colesterol $=148 ; \mathrm{HDL}$ $=38 ; \mathrm{LDL}=96 ; \mathrm{VLDL}=13,2$. Exame de urina tipo I: cristais de ác. úrico +++ ; Clearance de creatinina (urina de 24h): 49 ml/min/1,73m²; Proteinúria de 24h: 442 mg. Eletrocardiograma: ritmo sinusal, sobrecarga ventricular esquerda e radiografia de tórax: cardiomegalia. 


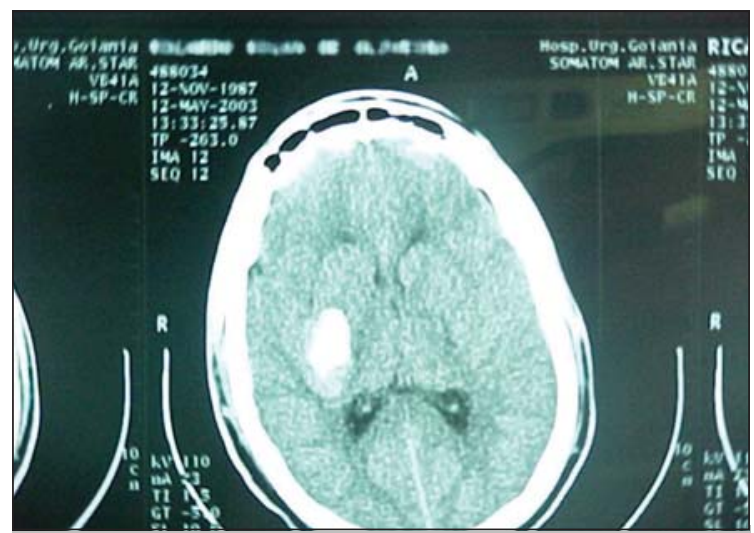

Fig. 1 - Tomografia computadorizada de crânio evidenciando hemorragia intraparenquimatosa e periventricular a esquerda

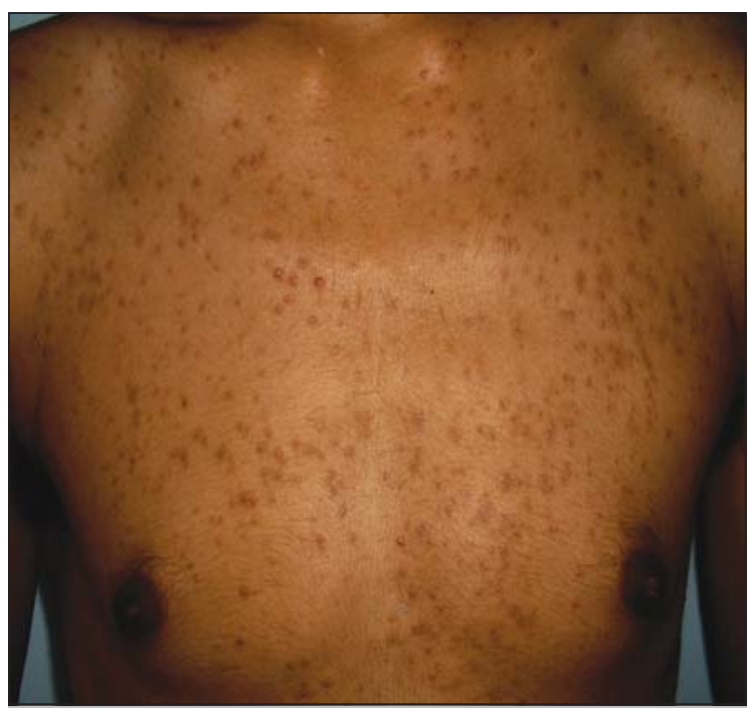

Fig. 2 - Acne em região anterior de tórax

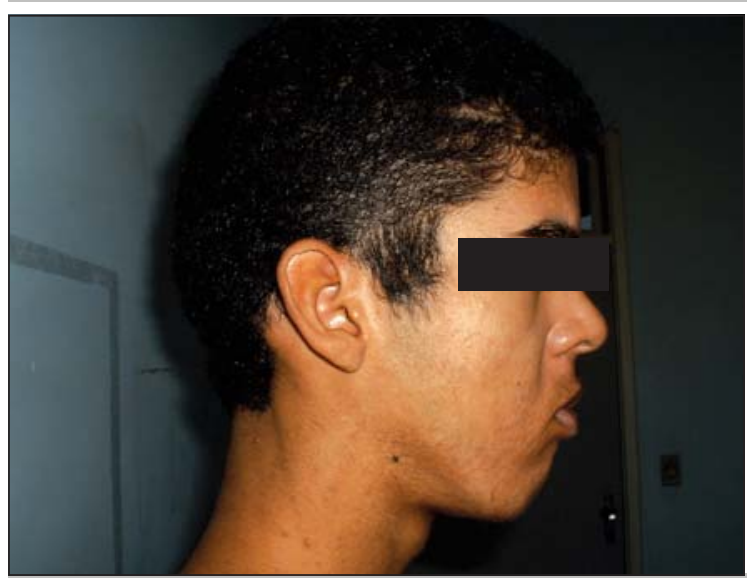

Fig. 3 - Paciente à admissão: acne em face

Evolução clínica - internado no setor de terapia intensiva por dez dias, recebendo nitroprussiato de sódio venoso pela maior parte do tempo, associado a antihipertensivos orais. Solicitado exames: ACTH: 498 (N < $60 \mathrm{pg} / \mathrm{ml}$ ); 11-deoxicortisol: 41 (N: 0 a 0,8 ng/ml); androstenediona: 28 ( $\mathrm{N}: 0,4$ a $2 \mathrm{ng} / \mathrm{ml})$; testosterona total: $789(\mathrm{~N}: 241$ a $827 \mathrm{ng} / \mathrm{dl})$; testosterona livre: 22 (N: 0,3 a $21 \mathrm{pg} / \mathrm{ml}$ ); 17-OH progesterona: 109 (N: 50 a $300 \mathrm{ng} / \mathrm{dl}$ ); DHEA-S: 421 ( $\mathrm{N}: 4$ a $68 \mathrm{mg} / \mathrm{dl}$ ); cortisol 8h: 3,5 ( $\mathrm{N}: 4,3$ a $25 \mathrm{mg} / \mathrm{dl}$ ) fechando o diagnóstico de hiperplasia supra-renal congênita por deficiência de $11-\beta$ hidroxilase. Prescritos dois anti-hipertensivos orais (nifedipina e enalapril), dexametasona $0,5 \mathrm{mg}$ oral/dia e fisioterapia motora além da dieta hipossódica.

Evoluiu com regressão total do quadro neurológico (sem seqüelas aparentes) e da acne, com manutenção razoável dos níveis pressóricos recebendo alta após quatorze dias do início do quadro.

\section{Discussão}

Este caso clínico nos mostra lesões de órgãosalvo, tanto agudas (distúrbio vascular hemorrágico) como crônicas (insuficiência renal crônica classe III), decorrentes de níveis pressóricos elevados sugerindo, causa secundária.

Devemos lembrar de hipertensão arterial (HAS) secundária em: início < 30 ou > 50 anos; HAS grave ou resistente à terapêutica; tríade de feocromocitoma; massas ou sopros abdominais; assimetria de pulsos femorais; creatinina sérica elevada; fármacos; hipopotassemia espontânea $\left(\mathrm{K}^{+}<3,0 \mathrm{mEq} / \mathrm{l}\right)$; EAS anormal (hematúria ou proteinúria); fácies (acromegalia, Cushing, hipertireoidismo) ${ }^{1}$.

Os sinais clínicos que sugeriam alterações hormonais como causa base de todo processo foram a presença de acne e pubarca em fase precoce, com ginecomastia denotando estímulo androgênico excessivo; associados a níveis pressóricos elevados e hipocalemia (sugestiva de excesso mineralocorticóide circulante).

Bioquímica da biossíntese esteróide adrenal - 0 cortisol é um hormônio sintetizado a partir do colesterol na zona fasciculada do córtex da adrenal. Esse processo requer cinco conversões enzimáticas (fig. 4). Na conversão de 11-deoxicortisol em cortisol há a participação de um subtipo da enzima $11 \beta$-hidroxilase, encontrada sob duas isoformas: CYP11B1 e CYP11B2. Essas isoenzimas são citocromos P450 mitocondriais localizados na face interna de suas membranas, sendo a isoenzima CYP11B1 responsável por esse mecanismo².

Deficiência de 11- $\beta$-hidroxilase - Hiperplasia adrenal congênita por incapacidade de síntese de cortisol em geral se apresenta clinicamente com sinais de excesso androgênico, como masculinização da genitália externa feminina ou puberdade precoce no sexo masculino.

Os primeiros relatos da deficiência dessa isoenzima aconteceram na década de $1950^{3,4}$. A deficiência de 11 $\beta$-hidroxilase constitui $5 \%$ a $8 \%$ das causas de hiperplasia adrenal congênita, com incidência de aproximadamente um caso a cada cem mil nascidos vivos ${ }^{5}$. 


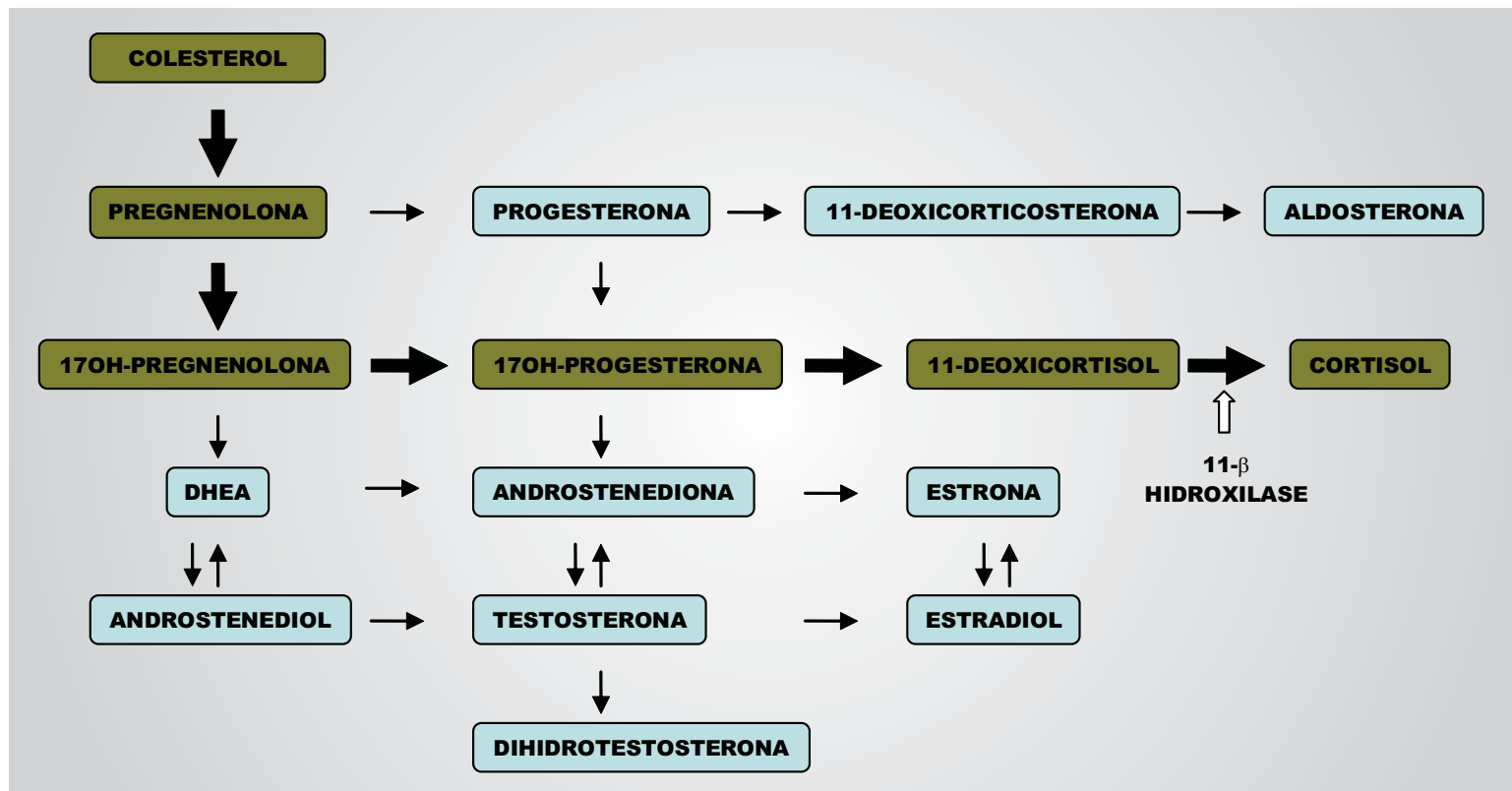

Fig. 4 - Esteroideogênese adrenal. Em destaque a via formadora do cortisol a partir do colesterol

Existem certos defeitos incomuns na esteroideogênese que resultam na síntese exagerada de precursores enzimáticos adrenais, especialmente a 11-desoxicortisol, induzindo a hipertensão por mineralocorticóides. A redução da produção de glicocorticóides resulta em um ineficaz feedback negativo no hipotálamo e pituitária anterior, causando secreção excessiva de ACTH. Esse estimula excessivamente o córtex da supra-renal com superprodução de precursores proximais do cortisol. Tais precursores possuem atividade mineralocorticóide, levando a elevação de níveis pressóricos, entre outras anormalidades.

Diagnóstico laboratorial - O diagnóstico específico da deficiência de 11- $\beta$-hidroxilase pode ser feito através de níveis basais altos de deoxicorticosterona e/ou11deoxicortisol séricos ou aumento da excreção de tetrahidrometabólitos desses compostos na urina de 24 horas. Tal diagnóstico deve ser suspeitado em pacientes com níveis séricos de ACTH elevados três vezes ou mais acima do percentil 95 previsto para a idade. Apesar de a maioria apresentar elevação de todos esses hormônios, uma pequena parcela pode apresentar elevação seletiva de apenas um deles.

Apresentação clínica - Hipertensão arterial: aproximadamente dois terços dos pacientes com diagnóstico de deficiência de 11- $\beta$-hidroxilase possuem hipertensão arterial $^{6}$, geralmente apresentando-se nos primeiros anos de vida. Apesar de a elevação dos níveis pressóricos ser leve a moderada na maioria dos casos, hipertrofia ventricular esquerda e/ou retinopatia hipertensiva tem sido observadas em um terço dos pacientes, além de mortes por acidente cerebrovascular. Outros sinais de excesso mineralocorticóide, como hipocalemia ou fraqueza muscular, ocorrem em uma minoria de pacientes e não são correlacionados com os níveis pressóricos. $A$ causa da hipertensão não é bem esclarecida. Talvez ocorra por excesso de deoxicorticosterona sérica ${ }^{5}$.

Perda de sal - Ainda que a hipertensão arterial seja o marco clássico da deficiência de 11- $\beta$-hidroxilase, ocasionalmente, durante a infância, alguns pacientes podem desenvolver sinais de deficiência mineralocorticóide, como hipocalemia, hiponatremia e hipovolemia. Em alguns casos, é explicado pela terapia glicocorticóide. Em outros, essa deficiência mineralocorticóide acontece antes do tratamento por mecanismos ainda não bem conhecidos, atribuídos à perda da sensibilidade periférica a esses metabólitos ${ }^{5}$.

Virilização - Recém-nascidos do sexo feminino portadores de deficiência de 11 - $\beta$-hidroxilase já apresentam algum grau de virilização ao nascimento. Esse processo inicia-se a partir da sexta semana de gestação. Clitoromegalia pode ser severa ao ponto de dificultar distinção com pênis ${ }^{6-8}$. Meato uretral e abertura genital se deslocam anteriormente e podem fundir-se no sino urogenital. Pode haver fusão dos grandes lábios, em casos graves, indiferenciáveis da bolsa escrotal masculina.

Em contraste com a genitália externa, gônadas e estruturas internas (tubas ovarianas, útero e cérvice) derivados dos ductos de Müller estão preservados, pois a substância que normalmente causa a involução dessas estruturas nos homens (fator inibidor mülleriano) não é produzido pelo ovário fetal.

Outros sinais de excesso andrógeno incluem rápido crescimento somático na infância e fechamento precoce da epífise do esqueleto (baixa estatura). Tal fato não foi observado no paciente relatado em razão da alta estatura de seus pais. Pode haver amenorréia ou baixa espermatogênese por disfunção do eixo hipotalâmicopituitário-gonadal, além de hirsutismo e acne. 
Tratamento - Terapia glicocorticóide: a administração glicocorticóide repõe déficit de cortisol e reduz a secreção de ACTH. Tal terapia deve ser avaliada pela observação da curva de crescimento, avanço da idade óssea, andrarca e mensuração de andrógenos precursores do cortisol como a 4-androstenediona. Hidrocortisona oral é a terapia de escolha na infância. Se já houver fechamento de epífises, o regime terapêutico pode ser mudado para prednisona ou dexametasona.

\section{REFERÊNCIAS}

1. Sociedade Brasileira de Hipertensão, Sociedade Brasileira de Cardiologia, Sociedade Brasileira de Nefrologia - Investigação clínico-laboratorial \& Decisão terapêutica - IV Diretrizes Brasileiras de Hipertensão Arterial, 2002, 1:8.

2. Yanagibashi K, Haniu M, Shively JE, Shen WH, Hall P. The synthesis of aldosterone by the adrenal cortex. J Biol Chem 1986; 261:355662.

3. Shepard TH, Clausen SW. Case of adrenogenital syndrome with hypertension treated with cortisone. Pediatrics 1951; 8:805-13.

4. Wilkins L, Gardner LI, Crigler JF. Further studies on the treatment of congenital adrenal hyperplasia with cortisone. J Clin Endocrinol Metab 1952; 12:1015-30.

5. Zachmann M, Tassinari D, Prader A. Clinical and biochemical variability of congenital adrenal hyperplasia due to 11 betahydroxylase deficiency. A study of 25 patients. J Clin Endocrinol Metab 1983; 561:222-9.
Terapia anti-hipertensiva - Espironolactona ou amlorida para corrigir hipocalemia e tratar hipertensão leve (não presentes neste caso). Se esses agentes não forem suficientes, ou na ausência desses, bloqueadores de canal de cálcio como nifedipina ou verapamil podem ser usados ${ }^{9}$.

O diagnóstico pré-natal pode ser realizado através da dosagem de 11-deoxicortisol no fluido amniótico ${ }^{10}$ e correção da genitália ambígua através de procedimento cirúrgico.

6. Rosler A, Leiberman E, Sack J et al. Clinical variabilitv of congenital adrenal hyperplasia due to 11 -beta-hydroxylase deficiency. Horm Res 1982; Ib:133-41.

7. Harinarayan $\mathrm{CV}$, Ammini AC, Karmarkar MG et al. Congenital adrenal hyperplasia and complete masculinization masquerading as sexual precocity and cryptorchidism. Indian Pediatr 1992; 29:103-6.

8. Bistritzer T, Sack J, Eshkol A, Zur H, Katznelson D. Sex reassignment in a girl with 11-beta-hydroxylase deficiency. Isr J Med Sci 1984; 20:55-8.

9. Marg A, Kuban RJ, Behlke J, Dettmer R, Ruckpaul K. Crystallization and X-ray examination of bovine adrenodoxin. J Mol Biol 1992; 227:945-7.

10. Rosler A, Weshler N, Leiberman Eet al. 11-Beta-hydroxylase deficiency congenital adrenal hyperplasia: update of prenatal diagnosis. J Clin Endocrinol Metab 1988; 66:830-8. 